\title{
Commentary \\ Saline volume expansion and cardiovascular physiology: novel observations, old explanations, and new questions
}

\author{
James L Robotham \\ Professor and Chairman, Department of Anesthesiology, University of Rochester, Rochester, New York, USA
}

Corresponding author: James L Robotham, james_robotham@urmc.rochester.edu

Published online: 1 September 2004

Critical Care 2004, 8:315-318 (DOI 10.1186/cc2944)

This article is online at http://ccforum.com/content/8/5/315

(c) 2004 BioMed Central Ltd

Related to Research by Kumar et al., see issue 8.3, page 201

\begin{abstract}
In a clinical investigation, Kumar and coworkers reported the hemodynamic events that accompany plasma volume expansion over 3 hours in healthy adult volunteers, and found that increases in stroke volume (SV) may be related to increases in left ventricular (LV)/right ventricular (RV) end-diastolic volume, as they expected, but also to decreases in LV/RV end-systolic volume. The latter finding suggests increased contractility and/or decreased afterload, which do not fit with their perception that clinicians ascribe increases in SV to increases in end-diastolic volume based on Starling's work. Increased ejection fraction and decreased vascular resistances were also observed. The same authors recently reported novel data suggesting that reduced blood viscosity may account for the observed reduction in vascular resistances with saline volume expansion. However, the variances in preload and afterload, along with uncertainty in estimates of contractility, substantially limit their ability to define a primary mechanism to explain decreases in LV end-systolic volume. A focus on using ejection fraction to evaluate the integrated performance of the cardiovascular system is provided to broaden this analytic perspective. Sagawa and colleagues described an approach to estimate the relationship, under clinical conditions, between ventricular and arterial bed elastances (i.e. maximal ventricular systolic elastance [Emax] and maximal arterial systolic elastance [Ea]), reflecting ventricular-arterial coupling. I used the mean data provided in one of the reports from Kumar and coworkers to calculate that LV Emax decreased from 1.09 to $0.96 \mathrm{mmHg} / \mathrm{ml}$ with saline volume expansion, while Ea decreased from 1.1 to $0.97 \mathrm{mmHg} / \mathrm{ml}$ and the $\mathrm{SV}$ increased (i.e. the increase in mean SV was associated with a decrease in mean afterload while the mean contractility decreased). The results reported by Kumar and coworkers invite further studies in normal and critically ill patients during acute saline-induced plasma volume expansion and hemodilution. If reduced viscosity decreases afterload, then this raises the questions by what mechanism, and what is the balance of benefit and harm associated with reduced blood viscosity affecting oxygen delivery? Why the mean Emax might decrease must be evaluated with respect to benefit in reducing ventricular work or a negative inotropic effect of saline.
\end{abstract}

Keywords afterload, cardiovascular physiology, contactility, hemodynamics, preload, ventricular function

The article by Kumar and coworkers [1] is one of a series of three papers $[2,3]$ by the authors addressing the acute hemodynamic events that accompany plasma volume expansion over 3-5 hours in healthy young adult volunteers. Taking into account all three reports, the most interesting finding is that increases in stroke volume (SV) following saline infusion over 3 hours may be variably related to increases in left ventricular (LV) end-diastolic volume (EDV) and/or decreases in LV end-systolic volume (ESV). The second interesting finding, from one of the reports [3],

$\mathrm{CO}=$ cardiac output; $\mathrm{Ea}=$ maximal arterial systolic elastance; $\mathrm{EDV}=$ end-diastolic volume; $\mathrm{EF}=$ ejection fraction; Emax $=$ maximal ventricular systolic elastance; ESV = end-systolic volume; LV = left ventricular; Pes = end-systolic pressure; RV = right ventricular; SV = stroke volume; Vo = zero Pes intercept of Emax from a pressure-volume diagram. 
involves novel data suggesting that the acute dilution by reducing blood viscosity may account for a substantial proportion of the reduction in vascular resistance in the systemic and pulmonary arterial beds, which was consistently observed at 3 hours, but diminished after the infusion was stopped and normal viscosity re-established. The authors did not investigate possible endothelial mechano-transduction mechanisms, or determine whether the reduction in systemic venous resistance that accompanied this decrease in viscosity might alter the time constant for venous return, thus enhancing cardiac output (CO).

The fundamental findings (albeit with substantial variability among the individuals studied) were that 3 I of saline infused over 3 hours increases the LVEDV, RVEDV, CO, SV and ejection fraction (EF), whereas the LVESV, and systemic and pulmonary vascular resistances decreased. Consistent with many previous reports, changes in RVEDV and LVEDV did not consistently correlate with changes in ventricular enddiastolic pressures [2], but the unexpectedly high central venous and pulmonary capillary wedge pressures reported [1-3] are consistent with and account for this finding [4]. Multiple measures of LV contractility, which are to varying degrees load dependent, suggest no change or an increase in ventricular contractile function. However, the variances in preload and afterload, along with the uncertainty in estimates of contractility, substantially limit the ability of the authors to draw any conclusions with respect to a primary mechanism underlying the decreases in LVESV.

My comments focus on the Frank-Starling mechanism, which the authors use as the basis for their argument that clinicians have incorrectly assumed that increases in SV and CO with plasma volume expansion were determined by an increase in the LVEDV. An a priori physiologic analysis of an increase in plasma volume may place the argument in a broader perspective. Otto Frank, in 1899, reported the first experimentally derived ventricular pressure-volume diagrams, emphasizing that the end-systolic pressure and volume are determined by events that occur in the immediately preceding cardiac cycle. Starling never plotted a pressure-volume relationship based on raw experimental data, although one of his students did [5]. Starling did plot: an end-systolic and end-diastolic isovolumic pressure-volume relationship from Frank's work; CO against mean right atrial pressure; and external work against EDV [5]. Starling's 'Law of the Heart' is frequently misunderstood in interpreting Starling's use of mean right atrial pressure as preload. Preload is now more accurately defined as the ventricular EDV. Thus, using relatively healthy isolated hearts with ability to control the mean arterial pressure, Starling found a strong correlation between the mean right atrial pressure and the SV, with small changes in afterload and heart rate being relatively unimportant. He did postulate that a larger ventricular volume permitted a larger chemically thus the SV, when the afterload was maintained relatively constant by experimental means.

Indeed, years later Sagawa and coworkers [5], using computer controlled isolated ventricles, demonstrated that the end-systolic pressure-volume relationship (Pes-ESV) was a straight line, reflecting the maximum ventricular systolic elastance (Emax) of the ventricle. Emax served as a load independent measure of contractility; as the EDV increased, both ESV and the SV would increase (Fig. 1). The degree to which the ESV and SV changed with increasing EDV could be altered by changing the afterload (or more precisely the impedance, incorporating arterial vascular resistance, compliance, and inertance) or contractility. Experimentally, with contractility constant while acutely increasing EDV, one could first reduce afterload to an extremely low value and then progressively increase the afterload to limit ejection. This would yield serial results showing the following: first a decreased ESV and increased SV; then an increased $\Delta S V=$ increased $\triangle E D V$; and finally an increased ESV = EDV with $S V=0$ when the afterload is sufficient to prevent ejection.

If one uses atrial pressure as a measure of preload (as did Starling), then the diastolic compliance of the ventricle would define the volume change, with pericardial constraint (when present) becoming the dominant factor defining the shape of the diastolic pressure-volume relationship $[4,6,7]$. Only by instantaneously controlling very precisely the vascular input impedance (afterload) and contractility during a single cardiac cycle will the increase in EDV equal the increase in SV [5]. To be noted in passing, radionuclide cineangiography findings in one of the reports from Kumar and coworkers [2] showed a statistically significant increase of $10 \%(8 \mathrm{ml})$ in LVEDV and RVEDV with acute volume loading, and a reasonable correlation of $\triangle S V$ respectively with $\triangle R V E D V$ and $\triangle$ LVEDV. The decrease in ESV in both ventricles was only $2 \mathrm{ml}$, and four out of 12 individuals exhibited decreases in RVEDV with volume loading. These findings suggest that ventricular interdependence is not a dominant factor among the physiologic mechanisms. However, the unexpected finding in most of these studies was that the average central venous pressure was approximately $9.5 \mathrm{mmHg}$ at baseline in supine individuals who had been NPO overnight, and increased to an average of $12.5 \mathrm{mmHg}$, while pulmonary capillary wedge pressure was $10 \mathrm{mmHg}$ at baseline and increased on average to $15 \mathrm{mmHg}$. This strongly suggests either that the zero calibration position was problematic or that the individuals' control states were moderately hypervolemic, further limiting extrapolation of the results to hypovolemic patients.

Perhaps the easiest way to evaluate the clinical implications of acute plasma volume loading in these studies is to consider the EF. This parameter is widely used, incorrectly by most, as a measure of ventricular function [8]. It is rather a fascinating parameter that integrates contractility, preload, 
Figure 1

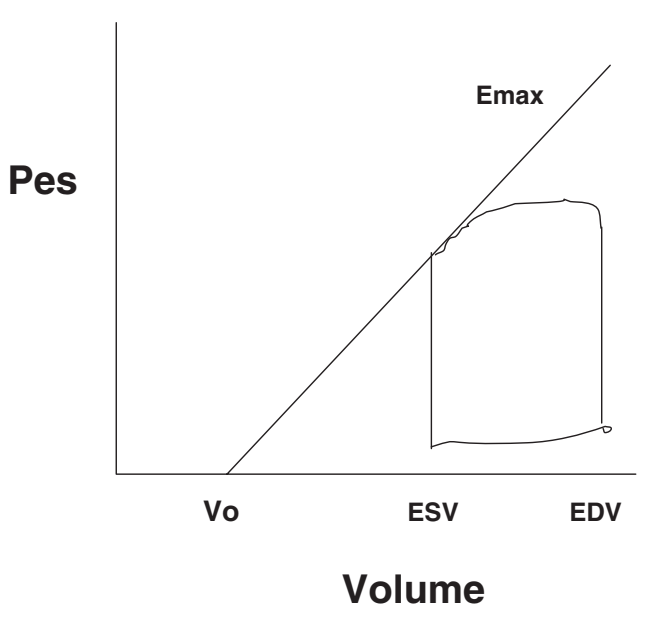

A simplified schematic of a single ventricular pressure-volume loop. EDV, end-diastolic volume; Emax, maximal ventricular systolic elastance; ESV, end-systolic volume; Pes, end-systolic pressure; Vo, zero Pes intercept of Emax from a pressure-volume diagram.

afterload, and Vo (the zero pressure intercept of the endsystolic pressure-volume relationship). If preload, afterload, and $V_{o}$ are all maintained constant, then a change in EF is a measure of ventricular contractility. However, mathematically, EF will increase if either EDV or Vo increase because of the following relationship:

$$
E F=([E D V-E S V] / E D V)-V o / E D V
$$

This equation can be rewritten as follows:

$$
E F=1-(P e s / E D V)(1 / E m a x)-(\text { Vo/EDV })
$$

Note that EF reflects the influences of preload (EDV), afterload (Pes), Vo (increases associated clinically with heart failure), and contractility (Emax). Thus, changes in any or all of the physiologic parameters that define EF can result in a myriad of combinations that preclude use of EF as a definitive measure of change in any one of them under clinical conditions. Indeed, the widespread application of EF as a clinical tool is precisely because it integrates all of these parameters into a single number that reflects the overall clinical state of the coupled ventricular-arterial systems [8]. However, it is clear that a decreased afterload and/or increased contractility must be present in the reports from Kumar and coworkers [1-3] for the LVESV to decrease and for SV and CO to increase.

Perhaps the most useful insight into use of EF from the reports from Kumar and coworkers is as follows; if EF remains relatively constant, even accepting a slight increase due to the mathematical consequence of increasing EDV, then the distribution of this increase will be proportional to EF (i.e. if $E F=0.6$, then $60 \%$ of the increase in $E D V$ will equal the increase in SV). Thus, one would predict increases in EDV, ESV, and SV. At the two extremes, if EF $=0.9$ and the EDV increases $10 \mathrm{ml}$, then $9 \mathrm{ml}$ will added to the SV and $1 \mathrm{ml}$ to the ESV. Conversely, if EF $=0.1$ and the EDV increases $10 \mathrm{ml}$, then $1 \mathrm{ml}$ will be added to the SV and $9 \mathrm{ml}$ to the ESV, assuming constant afterload and contractility. This raises the serious question as to whether one can generalize the findings of volume expansion in healthy volunteers to seriously ill patients who are variably hypovolumic and vasoconstricted, or septic and vasodilated with impaired contractility, given the variance in baseline conditions existing in myocardial and endothelial function as regulated by neural and humoral factors in addition to administered pharmaceutical agents in such patients. To my knowledge, the degree to which a change in viscosity would further affect afterload or venous return, when the mechanoreceptor function of the endothelium is markedly altered during an inflammatory state, has not been studied in the clinical setting.

Returning to the results of the three reports from Kumar and coworkers [1-3], including the one published in this journal, the critical question that remains unanswered is the primary mechanism that would explain a decrease in ESV that appears transiently during the time of maximum volume expansion and hemodilution with saline. The authors focus on increased EF, and in one report [3] measures of Pes/LVESV suggest that contractility has increased, although multiple other estimates of contractility exhibit no change. Overall, however, the largest consistent changes that they observe are increases in SV and CO with decreases in pulmonary and systemic vascular resistances. Although in many individuals preload increases would appear to account for much of the increase in SV, given little change in contractility and a decreased afterload, the series of reports lacks a rigorous evaluation to address the key questions directly.

It would seem possible that the authors and others who might replicate this study in normal or critically ill patients should turn to the classic book by Sagawa and coworkers published in 1988 [5]. Chapter 5 in that book describes a relatively straightforward approach to estimating the relationship under clinical conditions between ventricular and arterial bed elastances (i.e. Emax and Ea, reflecting ventricular-arterial coupling). Briefly, by plotting Emax and Ea with Pes versus SV on pressure-volume axes, the two straight lines - one with a negative slope and the other with a positive slope - must intersect at the point where a common Pes and SV occur (Fig. 2). I used mean data provided in a prior report from Kumar and coworkers [3] that allow this to be done. The results were that LV Emax decreased from 1.09 to $0.96 \mathrm{mmHg} / \mathrm{ml}$ with saline volume expansion, while Ea decreased from 1.1 to $0.97 \mathrm{mmHg} / \mathrm{ml}$ and the SV increased. Thus, in that cohort of 32 male volunteers in whom there was a statistically significant decrease in systemic vascular arterial resistance, the mean results are consistent with a decrease 
Figure 2

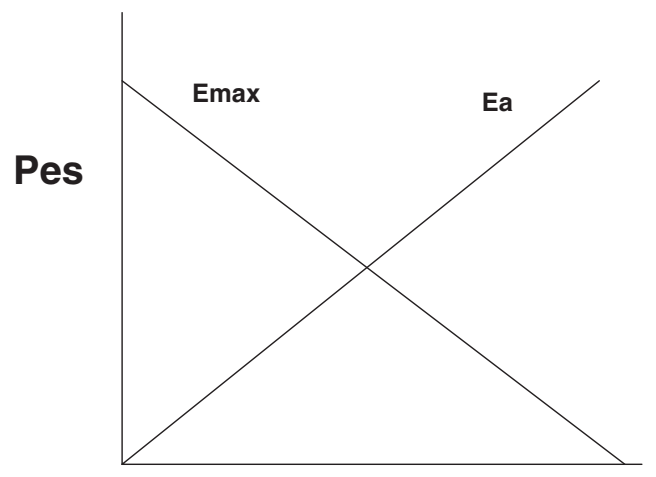

Stroke Volume

A simplified schematic of the relationship between Emax (maximal ventricular systolic elastance) and Ea (maximal arterial systolic elastance) with the intersection, defining the resulting stroke volume. Pes, end-systolic pressure.

in systemic afterload rather than an increase in contractility accounting for the increase in SV and decrease in ESV. Any statistical significance of this analysis is of course lacking until the authors derive Emax and Ea for each individual before and after volume expansion, and then perform a complete statistical analysis on all of the data.

Bringing this all back to the clinical application of EF [8], the relationship between Emax and Ea can be derived as follows:

$$
\mathrm{EF}=(\mathrm{Emax} /[\mathrm{Emax}+\mathrm{Ea}])(1-\mathrm{Vo} / \mathrm{EDV})
$$

This restates the conclusion that EF is an effective clinical measure because it provides a single number that is sensitive to changes in ventricular function, arterial impedance, and the poorly understood Vo relative to EDV.

In conclusion, the findings presented by Kumar and coworkers invite further studies in normal and critically ill patients during acute saline induced plasma volume expansion or hemodilution. Starling's Law was derived under highly controlled experimental circumstances, such that assumptions that volume expansion should increase SV by increases in preload alone in a clinical setting requires consideration of a far more complex (patho)physiological analysis. If Kumar and coworkers suggested mechanism of transient lowering of viscosity is correct, then this would explain a dominant role of reducing afterload on both right and left ventricles. It would also raise questions regarding the mechanism that is responsible and regarding the balance of benefit and harm associated with reduced blood viscosity affecting oxygen delivery. Why the mean Emax might decrease must be evaluated with respect to likely benefit in reducing ventricular work and oxygen consumption, or reflecting a negative inotropic effect of saline that is masked by the reduced afterload effect. Furthermore, the possible role played by reduced viscosity in the resistance to venous return could be a logical additional consequence if the same occurs on the arterial side. The door is open for clinicians to explore these temporal physiologic observations and many other related questions safely in a wide variety of normal and pathologic conditions.

\section{Competing interests}

The author declares that he has no competing interests.

\section{References}

1. Kumar A, Anel R, Bunnell E, Zanotti S, Habet K, Haery C, Marshall $S$, Cheang M, Neumann A, Ali A, et al.: Preload-independent mechanisms contribute to increased stroke volume following large volume saline infusion in normal volunteers: a prospective interventional study. Crit Care 2004, 8:R128-R136.

2. Kumar A, Anel R, Bunnell E, Habet K, Zanotti S, Marshall S Neumann A, Ali A, Cheang M, Kavinsky C, et al.: Pulmonary artery occlusion pressure and central venous pressure fail to predict ventricular filling volume, cardiac performance, or the response to volume infusion in normal subjects. Crit Care Med 2004, 32:691-699.

3. Kumar A, Anel R, Bunnell E, Habet K, Neumann A, Wolff D, Rosenson R, Cheang M, Parrillo JE: Effect of large volume infusion on left ventricular volumes, performance and contractility parameters in normal volunteers. Intensive Care Med 2004, 30:1361-1369.

4. Hamilton DR, Dani RS, Semlacher RA, Smith ER, Kieser TM, Tyberg JV: Effects of aortic constriction during experimental acute right ventricular pressure loading. Further insights into diastolic and systolic ventricular interaction. Circulation 1995 92:546-554.

5. Sagawa K, Maughan L, Suga H, Sunagawa K: Cardiac Contraction and the Pressure-Volume Relationship. Oxford: Oxford Press; 1988:3-41.

6. Takata M, Mitzner W, Robotham JL: Influence of the pericardium on ventricular loading during respiration. J App/ Physiol 1990, 68:1640-1650.

7. Takata M, Robotham JL: Ventricular external constraint by the lung and pericardium during positive end-expiratory pressure. Am Rev Respir Dis 1991, 143:872-875.

8. Robotham JL, Takata M, Berman M, Harasawa Y: Ejection fraction revisited. Anesthesiology 1991, 74:172-183. 